\title{
Accuracy Evaluation of a New Broad-band Vector Digital Spectrum Analyzer
}

\author{
Domenico Mirri", Gaetano Iuculano ${ }^{\S}$, Fabio Filicori ${ }^{\ddagger}$, Gaetano Pasini ${ }^{*}$, Pier Andrea Traverso ${ }^{\ddagger}$ \\ "Department of Electrical Engineering, University of Bologna, Viale Risorgimento 2, 40136 Bologna, Italy \\ Phone: +390512093472 Fax: +390512093470, Email: domenico.mirri@mail.ing.unibo.it \\ ${ }^{\ddagger}$ DEIS, University of Bologna, Viale Risorgimento 2, 40136 Bologna, Italy \\ Phone: +390512093035 Fax: +390512093073 \\ ${ }^{8}$ Dept. of Electronics and Telecommunications, Via Santa Marta, 3 - 50139 Firenze - Italy \\ Phone: +390554796276, Fax: +39055494569, Email: iuculano@ingfi1.ing.unifi.it
}

\begin{abstract}
In this paper the theoretical expression of the accuracy of a previously introduced vector digital spectrum analyzer, based on a random asynchronous sampling strategy whose bandwidth limitation is due uniquely to the bandwidth of the Sample/Hold circuit, has been deduced and compared with the simulated results. In order to avoid the influence of the conventional time origin and of the measuring instant on the result the ratio between each spectral component of the signal an its fundamental one elevated to the same order of the considered harmonic is introduced. It is shown that the asymptotic mean value of this new parameter is independent from the measurement occasion if the number of samples is sufficiently great; besides it is characterized by a bias which depends on the approximation of the estimated fundamental frequency of the signal with respect to its true value. At last a procedure to estimate the fundamental frequency is proposed.
\end{abstract}

\section{INTRODUCTION}

Recently we introduced a new vector spectrum analyzer [1] based on a random sampling strategy previously used for the realization of both a digital power meter $[2,3]$ and a power spectrum analyzer $[4,5]$. To this end it is necessary to recall that, according to the Fourier series, a generic spectral component $X_{n}$ of a periodic signal $x(t)$ :

$$
x(t)=\sum_{q=-M}^{+M} X_{q} \mathrm{e}^{\mathrm{j} 2 \pi q f_{1} t}
$$

where $f_{1}=1 / T_{1}$ is the fundamental frequency and $M$ the practically finite maximum order of harmonics, can be deduced by the well known relationship:

$$
X_{n}=\frac{1}{T_{\mathrm{I}}} \int_{-T_{1} / 2}^{+T_{1} / 2} x(t) \mathrm{e}^{-\mathrm{j} 2 \pi n f_{\mathrm{I}} \mathrm{d} t}
$$

For the digital implementation of this equation, the integral can be approximated by using a sequence of $2 N+1$ samples of the periodic signal and the estimate $\hat{X}_{n}$ of a generic spectral component can be expressed as follows:

$$
\hat{X}_{n}=\frac{1}{2 N+1} \sum_{i=p_{0}-N}^{p_{0}+N} x\left(t_{i}\right) e^{-\mathrm{j} 2 \pi f \tilde{f}_{1} t_{i}}
$$

where $p_{0}$ is a positive integer (greater than $N$ ) which marks the centred value of the sequence of $2 N+1$ samples, $\tilde{f}_{1}$ a suitable estimate of $f_{1}$ and $t_{i}$ a generic sampling instant. The adopted random sampling strategy is defined as:

$$
t_{i}=\left(i+Y_{i}\right) T_{s}+\tau
$$

where $T_{s}$ represents an "average" sampling rate, $Y_{i}$ a set of independent random variables with a probability density function uniformly distributed between $-1 / 2$ and $+1 / 2, \tau$ the unknown delay between the signal time origin and the sampling time generator. Therefore the estimator $\hat{X}_{n}$ becomes a random variable and, by substituting eqns. 1 and 4 into eqn.3, it can be expressed as follows:

$$
\hat{X}_{n}=h_{n n} X_{n} \mathrm{e}^{\mathrm{j} 2 \pi n\left(f_{1}-\tilde{f}_{1}\right)\left(\tau+p_{0} T_{x}\right)}+\sum_{\substack{q=-M \\ q \neq n}}^{+M} h_{q n} X_{q} \mathrm{e}^{\mathrm{j} 2 \pi\left(q f_{1}-n \tilde{f}_{i}\right)\left(\tau+p_{0} T_{x}\right)}
$$

where:

$$
h_{q n}=\frac{1}{2 N+1} \sum_{i=-N}^{+N} \mathrm{e}^{\mathrm{j} 2 \pi\left(q f_{i}-n \tilde{f}_{1}\right)\left(i+y_{\rho_{\mathrm{n}}+i}\right) T_{s}}
$$

This equation is valid also for $q=n$. We can also observe that $h_{q n}$ is independent from $\tau$. The expected value of $\hat{X}_{n}$ with respect to the random variables $\left\{Y_{p_{0}+i}\right\}$ results:

$$
\begin{aligned}
\mathrm{E}\left\{\hat{X}_{n}\right\}= & \mathrm{E}\left\{h_{n n}\right\} X_{n} \mathrm{e}^{\mathrm{j} 2 \pi n\left(f_{1}-\bar{f}_{1}\right)\left(\tau+p_{0} T_{s}\right)}+ \\
& +\sum_{\substack{q=-M \\
q \neq n}}^{+M} \mathrm{E}\left\{h_{q n}\right\} X_{q} \mathrm{e}^{\mathrm{j} 2 \pi\left(q_{1}-n \bar{f}_{1}\right)\left(\tau+p_{n} T_{s}\right)}
\end{aligned}
$$

where (see eqn.7 in [1]):

$$
\mathrm{E}\left\{h_{q n}\right\}=\operatorname{sinc}\left(\left(q f_{1}-n \tilde{f}_{1}\right)(2 N+1) T_{s}\right)
$$

is a real quantity, independent of $p_{0}$ since the random variables $\left\{Y_{p_{0}+i}\right\}$ have a common distribution for any $p_{0}$ and $i$, and coincides with the Fourier Transform of a continuous rectangular window of length $(2 N+1)$. By referring to the last expression of eqn.7 we observe the mean value of the estimate of $X_{n}$ is characterized by the following errors: a) an amplitude error due to $\mathrm{E}\left\{h_{n n}\right\} ;$ b) a phase error due to $\left.n\left(f_{1}-\tilde{f}_{1}\right)\left(\tau+p_{0} T_{s}\right) ; c\right)$ an error due to the influence of the other spectral components into the sum. Because eqn. 8 is not 
periodic, the aliasing effect is not present in this random sampling strategy and consequently the last error is due uniquely to the leakage effect

The expected value of $\hat{X}_{n}$ is conditioned to particular values of the delay $\tau$ and the centred point $p_{0}$ of the sampled values. By interpreting as random variables both $\tau$ and $p_{0}$, the unknown delay $\tau$ (eqn.4) can be assumed as a continuous variable uniformly distributed in a generic timeinterval $T^{\prime}$ and the centred value $p_{0}$ a discrete variable uniformly distributed in a generic discrete interval $(-m,+m)$. The random variables $\tau, p_{0}$ and $\left\{Y_{i}\right\}$ are mutually independent. In order to avoid the influence of the conventional time origin on the instrument performance and of the measuring instant, the asymptotic behaviour can be obtained by imposing that the quantities $T^{\prime}$ and/or $m$ are sufficiently large and theoretically tending to infinity. Obviously, when the period $T_{1}$ is known, the asymptotic behaviour can be obtained also if the time-interval $T^{\prime}$ coincides with $T_{1}$. The asymptotic expected value of $\hat{X}_{n}$ also with respect to these last quantities is expressed as in eqn.11 in [1]. From this equation we deduce that the asymptotic expected value is null. In order to overcome this cumbersome and also to avoid the phase error $n\left(f_{1}-\tilde{f}_{1}\right)\left(\tau+p_{0} T_{s}\right)$ we proposed to introduce the ratio $\hat{\rho}_{n}$ defined as follows [1]:

$$
\hat{\rho}_{n}=\frac{\hat{X}_{n}}{\left(\hat{X}_{1}\right)^{n}} \quad \text { for } n>1
$$

it can be shown that, on the hypothesis of a leakage effect sufficiently low, it results (see eqn.8):

$$
\underset{\rightarrow}{\mathrm{E}}\left\{\hat{\rho}_{n}\right\} \cong \frac{X_{n}}{X_{1}^{n}} \frac{\mathrm{E}\left\{h_{n n}\right\}}{\mathrm{E}^{n}\left\{h_{11}\right\}}=\frac{X_{n}}{X_{1}^{n}} \frac{\operatorname{sinc}(n \Delta)}{\operatorname{sinc}^{n}(\Delta)}
$$

where:

$$
\Delta=\left(f_{1}-\tilde{f}_{1}\right)(2 N+1) T_{s}
$$

From eqn.10 we observe that there is uniquely a relative amplitude error equal to $\operatorname{sinc}(n \Delta) / \operatorname{sinc}^{n}(\Delta)-1$. This error is null only if the condition $\Delta=0$, i.e. $\tilde{f}_{1}=f_{1}$, is satisfied; when this condition is only approximately verified, it is necessary to evaluate the relative amplitude error. To this end each term of the function $\operatorname{sinc}(n \Delta) / \operatorname{sinc}^{n}(\Delta)$ must be developed in Taylor series around the value $\Delta=0$. It can be shown that:

$$
\underset{\rightarrow}{\mathrm{E}}\left\{\hat{\rho}_{n}\right\} \cong \frac{X_{n}}{\left(X_{1}\right)^{n}} \frac{1-\frac{(\pi n \Delta)^{2}}{6}}{1-\frac{n(\pi \Delta)^{2}}{6}} \cong \frac{X_{n}}{\left(X_{1}\right)^{n}}(1+\varepsilon)
$$

where:

$$
\varepsilon=-n(n-1) \frac{(\pi \Delta)^{2}}{6}
$$

represents the relative amplitude error on the hypothesis that it is sufficiently low. For a prefixed value of $\varepsilon$, when the order $n$ of the estimated spectral component increases, the frequency of the signal must be estimated with greatest accuracy.

It can be shown that the asymptotic variance can be ex-

It allows to normalize for each $n$ the expected value $\mathrm{E}\left\{\hat{X}_{n}\right\}$ with respect to $\mathrm{E}\left\{\hat{X}_{1}\right\}$.

$$
\begin{aligned}
& \underset{\rightarrow}{\operatorname{Var}}\left\{\rho_{n}\right\} \cong \frac{1}{2 N+1}\left\{\frac{1}{\mathrm{E}^{2 n}\left\{h_{11}\right\}}\left[\sum_{q=-M}^{+M}\left(\frac{\left|X_{q}\right|}{\left|X_{1}\right|^{n}}\right)^{2}\left(1-\mathrm{E}_{0}^{2}\left\{h_{q n}\right\}\right)+(2 N+1) \sum_{\substack{q=-M \\
q \neq n}}^{+M}\left(\frac{\left|X_{q}\right|}{\left|X_{n}\right|}\right)^{2} E^{2}\left\{h_{q n}\right\}\right]\right. \\
& +n^{2} \frac{\mathrm{E}^{2}\left\{h_{n n}\right\}}{\mathrm{E}^{2(n+1)}\left\{h_{11}\right\}}\left[\sum_{q=-M}^{+M}\left[\frac{\left|X_{q}\right|}{\left|X_{1}\right|^{n}} \frac{\left|X_{n}\right|}{\left|X_{1}\right|}\right)^{2}\left(1-\mathrm{E}_{0}^{2}\left\{h_{q 1}\right\}\right)+(2 N+1) \sum_{\substack{q=-M \\
q \neq 1}}^{+M}\left(\frac{\left|X_{q}\right|}{\left|X_{1}\right|^{n}} \frac{\left|X_{n}\right|}{\left|X_{1}\right|}\right)^{2} E^{2}\left\{h_{q 1}\right\}\right] \\
& -2 n \frac{\mathrm{E}\left\{h_{n n}\right\}}{\mathrm{E}^{2 n+1}\left\{h_{11}\right\}} \frac{\left|X_{n}\right|^{2}}{\left|X_{1}\right|^{2 n}}\left[\mathrm{E}\left\{h_{(n-1)(n-1)}\right\}\left(1-\frac{\mathrm{E}_{0}\left\{h_{n n}\right\} \mathrm{E}_{0}\left\{h_{11}\right\}}{\mathrm{E}_{0}\left\{h_{(n-1)(n-1)}\right\}}\right)\right. \\
& \left.\left.+\sum_{\substack{q=-M \\
q \neq n}}^{+M}\left(\frac{X_{q} X_{q-n+1}^{*}}{X_{n} X_{1}^{*}}+\frac{X_{q}^{*} X_{q-n+1}}{X_{n}^{*} X_{1}}\right)\left(\mathrm{E}\left\{h_{(n-1)(n-1)}\right\}\left(1-\frac{\mathrm{E}_{0}\left\{h_{q n}\right\} \mathrm{E}_{0}\left\{h_{(q-n+1) 1}\right\}}{\mathrm{E}_{0}\left\{h_{(n-1)(n-1)}\right\}}\right)+\mathrm{E}\left\{h_{q n}\right\} E\left\{h_{(q-n+1) 1}\right\}(2 N+1)\right)\right]\right\}
\end{aligned}
$$

pressed as follows (eqn. A31):

\section{ACCURACY ANALYSIS}

The ratio $\hat{\rho}_{n}$, defined by eqn. 9 , represents the new output of the instrument. By evaluating the asymptotic mean of $\hat{\rho}_{n}$,
Taking into account eqns. 8 and 11 and recalling that $|n \Delta|$ must be sufficiently small in order to reduce the relative amplitude error (eqn.10), we can write:

$$
\begin{aligned}
& \mathrm{E}\left\{h_{q n}\right\} \cong \operatorname{sinc}\left[(q-n)(2 N+1) f_{1} T_{s}\right] \text { for } q \neq n \\
& \mathrm{E}\left\{h_{n n}\right\}=\operatorname{sinc}[n \Delta] \cong 1
\end{aligned}
$$




$$
\begin{aligned}
& \mathrm{E}_{0}\left\{h_{q n}\right\} \cong \operatorname{sinc}\left[(q-n) f_{1} T_{s}\right] \text { for } q \neq n \\
& \mathrm{E}_{0}\left\{h_{n n}\right\}=\operatorname{sinc}\left[n \frac{\Delta}{2 N+1}\right] \cong 1 .
\end{aligned}
$$

Obviously the previous approximations are acceptable for estimating the variance; indeed, for the evaluation of the amplitude error the effect of $n \Delta$ must be taken in more significant account. By substituting into eqn.14 we obtain the final result: which depend on the relative amplitude of the other spectral components.

Figure 1 shows the shape of the theoretical expression of $\operatorname{Var}_{\rightarrow}\left\{\rho_{n}\right\}$ (eqn.19) $X_{q}=1, N=50$ and $M=3$ respectively for $n=2$ (a) and $n=3$ (b). It is interesting to observe that the second term into eqn.19 predominates and tends to the value $n^{2}(M-1) /(2 N+1)$.

$$
\begin{aligned}
& \underset{\rightarrow}{\operatorname{Var}}\left\{\rho_{n}\right\} \cong \frac{1}{2 N+1}\left\{\sum_{\substack{q=-M \\
q \neq n}}^{+M}\left(\frac{\left|X_{q}\right|}{\left|X_{1}\right|^{n}}\right)^{2}\left[1-\operatorname{sinc}^{2}\left((q-n) f_{1} T_{s}\right)+(2 N+1) \operatorname{sinc}^{2}\left((q-n)(2 N+1) f_{1} T_{s}\right)\right]\right. \\
& +n^{2} \sum_{\substack{q=-M \\
q \neq 1}}^{+M}\left(\frac{\left|X_{q}\right|}{\left|X_{1}\right|^{n}} \frac{\left|X_{n}\right|}{\left|X_{1}\right|}\right)^{2}\left[1-\operatorname{sinc}^{2}\left((q-1) f_{1} T_{s}\right)+(2 N+1) \operatorname{sinc}^{2}\left((q-1)(2 N+1) f_{1} T_{s}\right)\right] \\
& \left.-n \sum_{\substack{q=-M \\
q * n}}^{+M}\left(\frac{X_{q} X_{q-n+1}^{*}}{X_{n} X_{1}^{*}}+\frac{X_{q}^{*} X_{q-n+1}}{X_{n}^{*} X_{1}}\right)\left[1-\operatorname{sinc}^{2}\left((q-n) f_{1} T_{s}\right)+(2 N+1) \operatorname{sinc}^{2}\left((q-n)(2 N+1) f_{1} T_{s}\right)\right]\right\}
\end{aligned}
$$

From this equation we deduce that the variance of the estimated ratio $\hat{\rho}_{n}$ decreases with a quantity which is inversely proportional to $(2 N+1)$, i.e. the number of samples of the window, while in the equally spaced one decreases with the square of it [3]; this quantity is multiplied by different terms
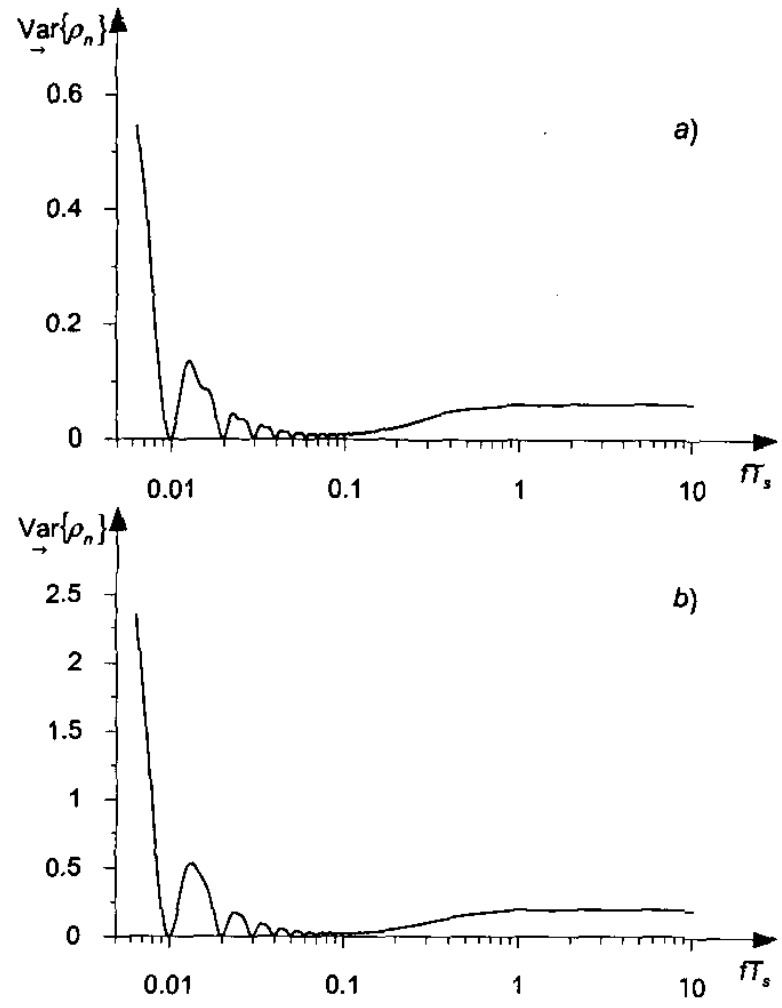

Fig. 1 - Shape of the theoretical expression of $\underset{\rightarrow}{\operatorname{Var}}\left\{\rho_{n}\right\}$ as a function of $\pi_{s}$

\section{SIMULATED RESULTS}

An estimate of the expected value of the ratio $\hat{\rho}_{n}$ of eqn.9 can be deduced by the following expression:

$$
\begin{aligned}
& \tilde{\mathrm{E}}\left\{\hat{\rho}_{n}\right\}=\frac{1}{m} \sum_{p_{0}=N+1}^{N+m} \frac{1}{R} \sum_{r=1}^{R} \frac{1}{W} \sum_{w=1}^{W}(2 N+1)^{n-1} . \\
& \frac{\sum_{i=p_{0}-N}^{p_{0}+N} x\left[\left(i+y_{i r}\right) T_{s}+\tau_{w} \mathrm{e}^{-\mathrm{j} 2 \pi \tilde{f}_{i}\left[\left(i+y_{i}\right) T_{s}+\tau_{w}\right]}\right.}{\left[\sum_{i=p_{0}-N}^{p_{0}+N} x\left[\left(i+y_{i r}\right) T_{s}+\tau_{w}\right] \mathrm{e}^{-\mathrm{j} 2 \pi \tilde{f}_{1}\left[\left(i+y_{s}\right) T_{s}+\tau_{w}\right]}\right]^{n}}
\end{aligned}
$$

where $y_{i r}$ is generic value of the random variable $Y_{i r}$, uniformly distributed in the interval between zero an one, $\tau_{w}$ is a generic value of the random variable $\tau$, uniformly distributed in a generic interval, for example between $-T^{\prime}$ and $+T^{\prime}$. In order to obtain the asymptotic value of this estimate, we can assume $T^{\prime}=\vec{T}_{1}$ because it is necessary to select $\tilde{f}_{1} \equiv f_{1}$ in order to have an amplitude error of the ratio $\hat{\rho}_{n}$ sufficiently small. From eqn. 20 we deduce that the value of $N$ must be selected sufficiently great in order to obtain a small asymptotic variance of the ratio $\hat{\rho}_{n}$ and not too great in order to have also a small amplitude error of the same ratio; for example we can impose $N=10^{4}$.

An estimate of the variance of the ratio $\hat{\rho}_{n}$ of eqn. 9 can be deduced by considering the last expression of eqn.A14:

$$
\tilde{\operatorname{Var}}\left\{\hat{\rho}_{n}\right\}=\tilde{\mathrm{E}}\left\{\hat{\rho}_{n} \hat{\rho}_{n}^{*}\right\}-\tilde{\mathrm{E}}\left\{\hat{\rho}_{n}\right\} \mathbb{E}\left\{\hat{\rho}_{n}^{*}\right\}=\tilde{\mathrm{E}}\left\{\left.\hat{\rho}_{n}\right|^{2}\right\}-\mid \tilde{\mathrm{E}}\left\{\hat{\rho}_{n}\right\}^{2}(21)
$$


To this end, by recalling eqn.18, we can deduce firstly the estimate of the expected value of the product $\hat{\rho}_{n} \hat{\rho}_{n}^{*}$ :

$$
\begin{aligned}
& \tilde{\mathrm{E}}\left\{\hat{\rho}_{n} \rho_{n}^{*}\right\}=\frac{1}{m} \sum_{p_{\mathrm{B}}=N+1}^{N+m} \frac{1}{R} \sum_{r=1}^{R} \frac{1}{W} \sum_{w=1}^{W}(2 N+1)^{2(n-1)} . \\
& \frac{\sum_{i=p_{0}-N}^{p_{0}+N} x\left[\left(i+y_{i r}\right) T_{s}+\tau_{w}\right] \mathrm{e}^{-\mathrm{j} 2 \pi n \tilde{f}_{1}\left[\left(i+y_{i r}\right) T_{s}+\tau_{w}\right]}}{\left[\sum_{i=p_{0}-N}^{p_{0}+N} x\left[\left(i+y_{i r}\right) T_{s}+\tau_{w}\right] \mathrm{e}^{-\mathrm{j} 2 \tilde{\tilde{f}}_{1}\left[\left(i+y_{i r}\right) T_{s}+\tau_{w}\right]}\right]^{n}} .
\end{aligned}
$$

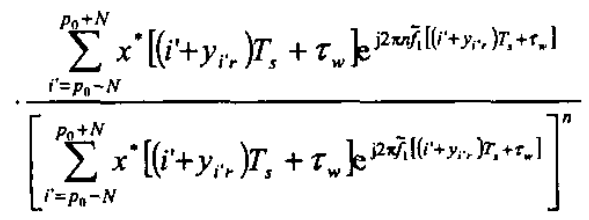

By introducing this equation into eqn.21 and by recalling eqn. 20 we obtain the final expression of the estimate of the variance. In order to consider the asymptotic properties of this variance, we must also in this case increase the value of $m$ till its value becomes a constant.

\section{CONCLUSION}

A vector spectrum analyzer based on a random asynchronous sampling strategy whose bandwidth limitation is due uniquely to bandwidth of the $\mathrm{S} / \mathrm{H}$ circuit is described. In order to obtain a measurement result independent of the measurement occasion, the ratio between each spectral component and the fundamental one is introduced. It has been shown that the mean value of this new parameter is independent from the measurement occasion if the number of samples is sufficiently large and it can be considered an unbiased estimate of the generic spectral component if the frequency of the fundamental frequency is sufficiently near to its true value. To this end a procedure for a software "locking" of this frequency is proposed.

\section{APPENDIX}

In order to separate the terms with $q=n$ and $q \neq n$ in $\hat{\rho}_{n}$ defined by eqn. 9 we introduce the parameter $g_{n}$ defined as follows:

$$
g_{n}=\sum_{\substack{q=-M \\ q \neq n}}^{+M} \frac{X_{q}}{X_{n}} \mathrm{e}^{\mathrm{j} 2 \pi(q-n) f_{1}\left(\tau+p_{0} \tau_{2}\right)} h_{q n}
$$

where $h_{q n}$ is given by eqn.6. This parameter takes into account the leakage effect, i.e. the effect of the other spectral components in the estimate of the $n^{\text {th }}$ one. Consequently, by recalling eqn.5, eqn.9 can be rewritten as follows:

$$
\hat{\rho}_{n}=\frac{X_{n}}{X_{1}^{n}} \frac{h_{n n}+g_{n}}{\left[h_{11}+g_{1}\right]^{n}}
$$

In order to evaluate the expected value of the parameter $g_{n}$ we have:

$$
\mathrm{E}\left\{g_{n}\right\}=\sum_{\substack{q=-M \\ q \neq n}}^{+M} \frac{X_{q}}{X_{n}} \mathrm{E}\left\{\mathrm{e}^{\mathrm{j} 2 \pi(q-n) f_{1} \tau} \mathrm{E}\left\{h_{q n} \mathrm{e}^{\mathrm{j} 2 \pi(q-n) f_{1} p_{0} T_{x}}\right\}\right.
$$

This equation takes into account the independence among $\tau, p_{0}$ and $\left\{Y_{i}\right\}$. Because both the unknown delay $\tau$ has been assumed random, uniformly distributed in a generic timeinterval $T^{\prime}$, and the centred value $p_{0}$ random, uniformly distribute in a generic discrete interval $(-m,+m)$, we obtain:

$$
\mathrm{E}\left\{g_{n}\right\}=\sum_{\substack{q=-M \\ q \neq n}}^{+M} \frac{X_{q}}{X_{n}} \operatorname{sinc}\left[(q-n) f_{1} T^{\prime}\right] \mathrm{E}\left\{h_{q n} \mathrm{e}^{\mathrm{j} 2 \mathrm{x}(q-n) f_{1} p_{0} T_{x}}\right\}
$$

In order to avoid the influence on the instrument performance both of the conventional time origin and of the measuring instant, the quantity $T^{\prime}$ must be sufficiently large and theoretically must tend to infinity, or, when the period $T_{1}$ of the periodic signal $x(t)$ is known, by imposing simply $T^{\prime}=k T_{1}$, with $k$ a positive integer. From eqn.A4 we deduce that the asymptotic mean is null for each $p_{0}$ :

$$
\underset{\mathbf{E}}{\mathrm{E}}\left\{g_{n}\right\}=0
$$

Therefore asymptotically the leakage effect nulls.

In order to deduce the statistical parameters of the ratio $\hat{\rho}_{n}$ it is convenient to introduce the complex random errors of $h_{n n}$ and $h_{11}$ with reference to their respective expected values $\mathrm{E}\left\{h_{n n}\right\}$ and $\mathrm{E}\left\{h_{11}\right\}$; therefore $\hat{\rho}_{n}$ of eqn.A2 can be rewritten as follows:

$$
\hat{\rho}_{n}=\frac{X_{n}}{X_{1}^{n}} \frac{\mathrm{E}\left\{h_{n n}\right\}+\Delta h_{n n}+g_{n}}{\left[\mathrm{E}\left\{h_{11}\right\}+\Delta h_{11}+g_{1}\right]^{n}}
$$

where the complex random error $\Delta h_{n n}$ is defined as follows:

$$
\Delta h_{n n}=h_{n n}-\mathrm{E}\left\{h_{n n}\right\}
$$

with $\mathrm{E}\left\{\Delta h_{n n}\right\}=0$. By assuming the variability of the amplitude error and the leakage effect sufficiently low so that at the denominator of eqn.A6 the successive powers $\left(\Delta h_{11}+g_{1}\right)^{n}$ with $n \geq 2$ can be neglected, we obtain:

$$
\hat{\rho}_{n} \cong \frac{X_{n}}{X_{1}^{n}} \frac{\mathrm{E}\left\{h_{n n}\right\}}{\mathrm{E}^{n}\left\{h_{11}\right\}} \frac{1+\frac{\Delta h_{n n}+g_{n}}{\mathrm{E}\left\{h_{n n}\right\}}}{1+n \frac{\Delta h_{11}+g_{1}}{\mathrm{E}\left\{h_{11}\right\}}}
$$

Now, by multiplying numerator and denominator of this ratio by the difference of the two denominator terms and, as usually, by neglecting the second order contributions of the errors we can write:

$$
\hat{\rho}_{n} \equiv \frac{X_{n}}{X_{1}^{n}} \frac{\mathrm{E}\left\{h_{n n}\right\}}{\mathrm{E}^{n}\left\{h_{11}\right\}}\left(1+\delta_{n}\right)
$$

where:

$$
\delta_{n}=\frac{\Delta h_{n n}+g_{n}}{\mathrm{E}\left\{h_{n n}\right\}}-n \frac{\Delta h_{11}+g_{1}}{\mathrm{E}\left\{h_{11}\right\}}
$$


It is useful to verify that $\delta_{1}=0$ because, for $n=1$, eqn. 9 becomes equal to one.

By considering the expected value of $\hat{\rho}_{n}$, we have:

$$
\mathrm{E}\left\{\hat{\rho}_{n}\right\} \cong \frac{X_{n}}{X_{1}^{n}} \frac{\mathrm{E}\left\{h_{n n}\right\}}{\mathrm{E}^{n}\left\{h_{11}\right\}}\left(1+\mathrm{E}\left\{\delta_{n}\right\}\right)
$$

where:

$$
\mathrm{E}\left\{\delta_{n}\right\}=\frac{\mathrm{E}\left\{g_{n}\right\}}{\mathrm{E}\left\{h_{n n}\right\}}-n \frac{\mathrm{E}\left\{g_{1}\right\}}{\mathrm{E}\left\{h_{11}\right\}}
$$

with $\mathrm{E}\left\{g_{n}\right\}$ given by eqn.A4 and $\mathrm{E}\left\{h_{n n}\right\}$ by eqn.8 with $q=n$. From eqn.A5 we deduce that asymptotically $\mathrm{E}\left\{\delta_{n}\right\}$ nulls:

$$
\underset{\mathrm{E}}{\rightarrow}\left\{\delta_{n}\right\}=0
$$

Therefore the asymptotic expected value of $\hat{\rho}_{n}$ results:

$$
\underset{\rightarrow}{\mathrm{E}}\left\{\hat{\rho}_{n}\right\} \cong \frac{X_{n}}{X_{1}^{n}} \frac{\mathrm{E}\left\{h_{n n}\right\}}{\mathrm{E}^{n}\left\{h_{11}\right\}}
$$

From this equation we deduce that asymptotically both the leakage and the phase error have no effect on the estimate of the parameter $\hat{\rho}_{n}$. Taking into account eqn.A14, eqn.A9 can be rewritten as follows:

$$
\hat{\rho}_{n} \cong \mathrm{E}\left\{\hat{\rho}_{n}\right\}\left(1+\delta_{n}\right)
$$

In order to evaluate the variance of the estimator $\hat{\rho}_{n}$, it is necessary to take into account that it is a complex quantity and therefore defined as follows:

$$
\operatorname{Var}\left\{\hat{\rho}_{n}\right\}=\mathrm{E}\left\{\hat{\rho}_{n} \hat{\rho}_{n}^{*}\right\}-\mathrm{E}\left\{\hat{\rho}_{n}\right\} \mathrm{E}\left\{\hat{\rho}_{n}^{*}\right\}
$$

By using the first expression of the variance, from eqns.A9 and $\mathrm{A} 11$ we deduce:

$$
\operatorname{Var}\left\{\hat{\rho}_{n}\right\} \cong \frac{\left|X_{n}\right|^{2}}{\left|X_{1}\right|^{2 n}} \frac{\mathrm{E}^{2}\left\{h_{n n}\right\}}{\mathrm{E}^{2 n}\left\{h_{11}\right\}}\left[\mathrm{E}\left\{\delta_{n} \delta_{n}^{*}\right\}-\mathrm{E}\left\{\delta_{n}\right\} \mathrm{E}\left\{\delta_{n}^{*}\right\}\right]
$$

By taking into account eqn.A13, the asymptotic value of this variance results:

$$
\underset{\rightarrow}{\operatorname{Var}}\left\{\hat{\rho}_{n}\right\}=\frac{\left|X_{n}\right|^{2}}{\left|X_{1}\right|^{2 n}} \frac{\mathrm{E}^{2}\left\{h_{n n}\right\}}{\mathrm{E}^{2 n}\left\{h_{11}\right\}} \mathrm{E}\left\{\delta_{n} \delta_{n}^{*}\right\}
$$

By recalling eqn.A10 where $\mathrm{E}\left\{h_{n n}\right\}$ is real (see eqn. 8 for $q=n)$, we can write:

$$
\begin{aligned}
& \delta_{n} \delta_{n}^{*}=\frac{\left(\Delta h_{n n}+g_{n}\right)\left(\Delta h_{n n}^{*}+g_{n}^{*}\right)}{\mathrm{E}^{2}\left\{h_{n n}\right\}}+n^{2} \frac{\left(\Delta h_{11}+g_{1}\right)\left(\Delta h_{11}^{*}+g_{1}^{*}\right)}{\mathrm{E}^{2}\left\{h_{11}\right\}}+ \\
& -\frac{n}{E\left\{h_{n n}\right\} E\left\{h_{11}\right\}}\left[\left(\Delta h_{n n}+g_{n}\right)\left(\Delta h_{11}^{*}+g_{1}^{*}\right)+\left(\Delta h_{n n}^{*}+g_{n}^{*}\right)\left(\Delta h_{11}+g_{1}\right)\right]
\end{aligned}
$$

In order to deduce the expected value of $\delta_{n} \delta_{n}^{*}$ we must therefore evaluate the contributions of the successive terms of this equation. To this end, in analogy to eqn.A7, in general we impose:

$$
\Delta h_{q n}=h_{q n}-\mathrm{E}\left\{h_{q n}\right\}
$$

where $E\left\{\Delta h_{q n}\right\}=0$. Besides, in analogy to eqn.A16 it results:

$$
\mathrm{E}\left\{\Delta h_{q n} \Delta h_{q n}^{*}\right\}=\operatorname{Var}\left\{h_{q n}\right\}=\mathrm{E}\left\{h_{q n} h_{q n}^{*}\right\}-\mathrm{E}^{2}\left\{h_{q n}\right\}
$$
being $\mathrm{E}\left\{h_{q n}\right\}$ real. On the other hand, by recalling eqn.6 we can evaluate the expected value conditioned to a particular value of $p_{0}$; that is:

$$
\begin{aligned}
& \mathrm{E}\left\{h_{q n} h_{q n}^{*} / p_{0}\right\}= \\
& =\frac{1}{2 N+1}+\frac{\operatorname{sinc}^{2}\left[\left(q f_{1}-n \tilde{f_{1}}\right) T_{s}\right]}{(2 N+1)^{2}} \sum_{\substack { i=-N \\
\begin{subarray}{c}{i \neq i=-N \\
i \neq i^{\prime}{ i = - N \\
\begin{subarray} { c } { i \neq i = - N \\
i \neq i ^ { \prime } } }\end{subarray}}^{+N} \mathrm{e}^{\mathrm{j} 2 \pi\left(q_{1}-n \tilde{f}_{i}\right)\left(i_{i-i^{\prime}}\right) T_{s}}= \\
& =\mathrm{E}\left\{h_{q n} h_{q n}^{*}\right\}
\end{aligned}
$$

where in the second passage we have taken into account the independence between $Y_{p_{0}+i}$ and $Y_{p_{0}+i^{\prime}}$ for $i \neq i^{\prime}$, while in the third passage we recalled eqn. 8 in [1], and in the last passage we point out that the conditioned expected value is independent from $p_{0}$. Moreover we can write:

$$
\begin{gathered}
\frac{1}{(2 N+1)^{2}} \sum_{i=-N}^{+N} \sum_{i \neq=-N}^{+N} \mathrm{e}^{\mathrm{j} 2 \pi\left(\phi_{i}^{\prime}\left(f_{1}-n \overline{n_{i}}\right)\left(i-i^{\prime}\right) T_{x}\right.}= \\
=\frac{\mathrm{E}^{2}\left\{h_{q n}\right\}}{\mathrm{E}_{0}^{2}\left\{h_{11}\right\}}-\frac{1}{2 N+1}
\end{gathered}
$$

where in the last passage we have taken into account eqn. 9 in [1] and eqn.8; besides we have introduced the symbol $E_{0}$ to indicate the expected value of eqn. 8 when $N=0$ :

$$
\mathrm{E}_{0}\left\{h_{q n}\right\}=\operatorname{sinc}\left(\left(q f_{1}-n \tilde{f}_{1}\right) T_{s}\right)
$$

By substituting into eqn.A22, we obtain:

$$
\mathrm{E}\left\{h_{q n} h_{q n}^{*}\right\}=\frac{1}{2 N+1}\left[1-\mathrm{E}_{0}^{2}\left\{h_{q n}\right\}+(2 N+1) \mathrm{E}^{2}\left\{h_{q n}\right\}\right]
$$

Therefore, from eqn.A21 it follows:

$$
\mathrm{E}\left\{\Delta h_{q n} \Delta h_{q n}^{*}\right\}=\frac{1}{2 N+1}\left[1-\mathrm{E}_{0}^{2}\left\{h_{q n}\right\}\right]
$$

which is a real quantity.

Further, by recalling eqn.A1 we have:

$$
g_{n} g_{n}^{*}=\sum_{\substack{q=-M \\ q \neq n}}^{+M} \sum_{\substack{q=-M \\ q \neq n}}^{+M} \frac{X_{q}}{X_{n}} \frac{X_{q^{\prime}}^{*}}{X_{n}^{*}} \mathrm{e}^{\mathrm{j} 2 \pi\left(q-q^{\prime}\right) f_{1}\left(\tau+p_{0} T_{s}\right)} h_{q n} h_{q^{\prime} n}^{*}
$$

As usually, we interpret the unknown delay $\tau$ and the centred value $p_{0}$ as random variables. The expected value of $g_{n} g_{n}^{*}$ must therefore be evaluated with respect both to the random vector $\left\{Y_{i}\right\}$ and these random variables; it results:

$$
\mathrm{E}\left\{g_{n} g_{n}^{*}\right\}=\sum_{\substack{q=-M \\ q \neq n}}^{+M} \sum_{\substack{q^{\prime}=-M \\ q^{\prime} \neq n}}^{+M} \frac{X_{q}}{X_{n}} \frac{X_{q^{\prime}}^{*}}{X_{n}^{*}} \mathrm{E}\left\{h_{q n} h_{q^{\prime} n}^{*} \mathrm{e}^{\mathrm{j} 2 \pi\left(q-q^{\prime}\right) f_{1} p_{0} T_{x}}\right\} \mathrm{E}\left\{\mathrm{e}^{\mathrm{j} 2 \pi\left(q-q^{\prime}\right) f_{1} \tau}\right\}
$$

whose asymptotic value with respect to $\tau\left(T^{\prime} \rightarrow \infty\right.$ or $T^{\prime}=k T_{1}$ when $T_{1}$ is known) is always null except for $q=q^{\prime}$. Therefore, by recalling eqn. A25 we have: 


$$
\underset{\rightarrow}{\mathrm{E}}\left\{g_{n} g_{n}^{*}\right\}=\frac{1}{2 N+1} \sum_{\substack{q=-M \\ q \neq n}}^{+M} \frac{\left|X_{q}\right|^{2}}{\left|X_{n}\right|^{2}}\left[1-\mathrm{E}_{0}^{2}\left\{h_{q n}\right\}+(2 N+1) \mathrm{E}^{2}\left\{h_{q n}\right\}\right]
$$

By considering all the terms of eqn.A19 it can be shown that the asymptotic mean value of $\delta_{n} \delta_{n}^{*}$ results:

$$
\begin{aligned}
& \underset{\rightarrow}{\mathrm{E}}\left\{\delta_{n} \delta_{n}^{*}\right\}=\frac{\mathrm{E}\left\{\Delta h_{n n} \Delta h_{n n}^{*}\right\}+\underset{\rightarrow}{\mathrm{E}}\left\{g_{n} g_{n}^{*}\right\}}{\mathrm{E}^{2}\left\{h_{n n}\right\}}+n^{2} \frac{\mathrm{E}\left\{\Delta h_{11} \Delta h_{11}^{*}\right\}+\underset{\rightarrow}{\mathrm{E}}\left\{g_{1} g_{1}^{*}\right\}}{\mathrm{E}^{2}\left\{h_{11}\right\}}-n \frac{2 \mathrm{E}\left\{\Delta h_{n n} \Delta h_{11}^{*}\right\}+\underset{\rightarrow}{\mathrm{E}}\left\{g_{n} g_{1}^{*}\right\}+\underset{\rightarrow}{\mathrm{E}}\left\{g_{n} g_{1}^{*}\right\}}{\mathrm{E}\left\{h_{n n}\right\} \mathrm{E}\left\{h_{11}\right\}}= \\
& =\frac{1}{2 N+1}\left\{\frac { 1 } { E ^ { 2 } \{ h _ { n n } \} } \left[\sum_{q=-M}^{+M}\left(\frac{\left|X_{q}\right|}{\left|X_{n}\right|}\right)^{2}\left(1-\mathrm{E}_{0}^{2}\left\{h_{q n}\right\}+(2 N+1) \sum_{\substack{q=-M \\
q \neq n}}^{+M}\left(\frac{\left|X_{q}\right|}{\left|X_{n}\right|}\right)^{2} \mathrm{E}^{2}\left\{h_{q n}\right\}\right]\right.\right. \\
& +\frac{n^{2}}{\mathrm{E}^{2}\left\{h_{11}\right\}}\left[\sum_{q=-M}^{+M}\left(\frac{\left|X_{q}\right|}{\left|X_{1}\right|}\right)^{2}\left(1-\mathrm{E}_{0}^{2}\left\{h_{q 1}\right\}\right)+(2 N+1) \sum_{\substack{q=-M \\
q \neq 1}}^{+M}\left(\frac{\left|X_{q}\right|}{\left|X_{1}\right|}\right)^{2} \mathrm{E}^{2}\left\{h_{q 1}\right\}\right] \\
& -\frac{n}{\mathrm{E}\left\{h_{n n}\right\} \mathrm{E}\left\{h_{11}\right\}}\left[2 \mathrm{E}\left\{h_{(n-1)(n-1)}\right\}\left(1-\frac{\mathrm{E}_{0}\left\{h_{n n}\right\} \mathrm{E}_{0}\left\{h_{11}\right\}}{\mathrm{E}_{0}\left\{h_{(n-1)(n-1)}\right\}}\right)\right. \\
& \left.\left.+\sum_{\substack{q=-M \\
q \neq n}}^{+M}\left(\frac{X_{q} X_{q-n+1}^{*}}{X_{n} X_{1}^{*}}+\frac{X_{q}^{*} X_{q-n+1}}{X_{n}^{*} X_{1}}\right)\left(\mathrm{E}\left\{h_{(n-1)(n-1)}\right\}\left(1-\frac{\mathrm{E}_{0}\left\{h_{q n} \mathrm{E}_{0}\left\{h_{(q-n+1))}\right\}\right.}{\mathrm{E}_{0}\left\{h_{(n-1)(n-1)}\right\}}\right)+(2 N+1) \mathrm{E}\left\{h_{q n}\right\} \mathrm{E}\left\{h_{(q-n+1) !}\right\}\right)\right]\right\}
\end{aligned}
$$

It is important to emphasize that the variance becomes null for $n=1$ since, from eqn.9, $\hat{\rho}_{1}=1$.

By substituting into eqn.A18 we obtain the final result:

$$
\begin{aligned}
& \underset{\rightarrow}{\operatorname{Var}}\left\{\rho_{n}\right\} \cong \frac{1}{2 N+1}\left\{\frac{1}{\mathrm{E}^{2 n}\left\{h_{11}\right\}}\left[\sum_{q=-M}^{+M}\left(\frac{\left|X_{q}\right|}{\left|X_{1}\right|^{n}}\right)^{2}\left(1-\mathrm{E}_{0}^{2}\left\{h_{q n}\right\}\right)+(2 N+1) \sum_{\substack{q=-M \\
q \neq n}}^{+M}\left(\frac{\left|X_{q}\right|}{\left|X_{n}\right|}\right)^{2} \mathrm{E}^{2}\left\{h_{q n}\right\}\right]\right. \\
& +n^{2} \frac{\mathrm{E}^{2}\left\{h_{n n}\right\}}{\mathrm{E}^{2(n+1)}\left\{h_{11}\right\}}\left[\sum_{q=-M}^{+M}\left(\frac{\left|X_{q}\right|}{\left|X_{1}\right|^{n}} \frac{\left|X_{n}\right|}{\left|X_{1}\right|}\right)^{2}\left(1-\mathrm{E}_{0}^{2}\left\{h_{q 1}\right\}+(2 N+1) \sum_{\substack{q=-M \\
q \neq 1}}^{+M}\left(\frac{\left|X_{q}\right|}{\left|X_{1}\right|^{n}} \frac{\left|X_{n}\right|}{\left|X_{1}\right|}\right)^{2} \mathrm{E}^{2}\left\{h_{q 1}\right\}\right]\right. \\
& -2 n \frac{\mathrm{E}_{q}\left\{h_{n n}\right\}}{\mathrm{E}^{2 n+1}\left\{h_{11}\right\}} \frac{\left|X_{n}\right|^{2}}{\left|X_{1}\right|^{2 n}}\left[\mathrm{E}\left\{h_{(n-1)(n-1)}\right\}\left(1-\frac{\mathrm{E}_{0}\left\{h_{n n}\right\} \mathrm{E}_{0}\left\{h_{11}\right\}}{\mathrm{E}_{0}\left\{h_{(n-1)(n-1)}\right\}}\right)\right. \\
& \left.+\sum_{q=-M}^{+M}\left(\frac{X_{q} X_{q-n+1}^{*}}{X_{n} X_{1}^{*}}+\frac{X_{q}^{*} X_{q-n+1}}{X_{n}^{*} X_{1}}\right)\left(\mathrm{E}\left\{h_{(n-1)(n-1)}\left\{1-\frac{\mathrm{E}_{0}\left\{h_{q n}\right\} \mathrm{E}_{0}\left\{h_{(q-n+1) 1}\right\}}{\mathrm{E}_{0}\left\{h_{(n-1)(n-1)}\right\}}\right)+(2 N+1) \mathrm{E}\left\{h_{q n}\right\} \mathrm{E}\left\{h_{(q-n+1) 1}\right\}\right)\right]\right\}
\end{aligned}
$$

\section{REFERENCES}

[1] D. Mirri, G. Iuculano, F. Filicori, G. Pasini: A broad-band digital spectrum analyzer based on a random asynchronous sampling strategy. IEEE Instrumentation and Measurement Technology Conference, Anchorage, Alaska, May 2002, pp. 1229-1233.
[4] D. Miri, G. luculano, G. Pasini, F. Filicori, L. Peretto: A Broad-Band Power Spectrum Analyzer Based On Twin-Channel Delayed Sampling. IEEE Trans. on Instrumentation and Measurement, vol.47, No.5, October 1998, pp.1346-1354.

[5] G. Pasini, D. Mirri, G. Iuculano, F, Filicori: Implementation and Performance Evaluation of a Broad-Band Power Spectrum Analyzer. IEEE Transactions on Instrumentation and Measurement, vol.50, No.4, August 2001, pp.1024-1029. 